\section{Polypharmacy management programmes: the SIMPATHY Project}

\author{
Alpana Mair, ${ }^{1}$ Fernando Fernandez-Llimos, ${ }^{2}$ SIMPATHY Consortium
}

Owing to advances in pharmacotherapy, older people are increasingly likely to be prescribed multiple medicines. Prescribing is largely based on single-disease evidencebased guidance, which does not generally take account of multimorbidity, which is now the norm in those over 65 years of age. ${ }^{1}$ Consequently, patients are prescribed several medicines recommended by disease-specific guidelines, which is potentially harmful for this specific population and challenging for them to manage. Inappropriate polypharmacy and medication non-adherence are two key inter-related problems in elderly multimorbid patients, with negative effects on health outcomes. Multimorbidity is associated with increased use of healthcare services (twice as high as nonmultimorbid) and hospitalisation (three times higher). ${ }^{2}$

'Polypharmacy', the prescribing of multiple medicines, has been described as one of the most pressing prescribing challenges. ${ }^{3}$ Among Scottish patients with two clinical conditions, $20.8 \%$ were receiving four to nine medicines, and $10.1 \%$ were receiving 10 or more medicines. ${ }^{4}$ Polypharmacy increases the likelihood of adverse effects, impacting significantly on health outcomes and healthcare resources. ${ }^{5}$ Notably, most adverse effects are preventable through appropriate medicine selection, monitoring and review. ${ }^{6}$ Such effects in older people may arise from many inter-related factors including: age-related physiological changes (eg, reduced renal and hepatic function); multimorbidities; prescribing of multiple medicines; and confusion over medicine taking. While, traditionally, polypharmacy has been described as prescribing of four or five medicines, it has been suggested that, given the emphasis on evidencebased practice, there should be a change in focus from inappropriate polypharmacy (inappropriate prescribing of too many medicines) to optimal polypharmacy

\footnotetext{
${ }^{1}$ Healthcare Quality and Strategy Directorate, Scottish Government I Edinburgh Scotland, Edinburgh, UK;

${ }^{2}$ Faculty of Pharmacy, Institute for Medicines Research (iMed.ULisboa), University of Lisboa, Lisboa, Portugal

Correspondence to Alpana Mair, Healthcare Quality and Strategy Directorate, Scottish Government, Edinburgh EH1 3DG UK; Alpana.Mair@gov.scot
}

(appropriate prescribing of many medicines). ${ }^{7}$ It is commonly accepted among healthcare providers that polypharmacy is an issue. However, an agreed concept and definition of polypharmacy does not exist.

A person with multiple morbidities usually requires multiple medicines to control his/her conditions. Some medical conditions, such as cardiovascular disease, require a number of prescribed medicines that exceeds the threshold of numeric definitions of polypharmacy. This uncertainty led some researchers to abandon the quantitative-based definition and adopt a qualitative-based one, coining the term 'inappropriate polypharmacy', accepting that some polypharmacy may be appropriate. $^{8}$

However, we should not ignore the fact that the consequences of polypharmacy are produced simultaneously by two different aspects of the use of multiple medicines: concomitant use of medicines, often prescribed by different physicians and non-medical prescribers (including self-medication by the patient and or carers), increases the chance of drug-drug interactions, duplication and adverse effects, constituting inappropriate prescribing. ${ }^{9}$ However, even with the most appropriate prescription process, increasing the number of medicines prescribed for a patient increases medication regimen complexity, which is a major driver of non-adherence. ${ }^{10}$

To reduce the negative consequences of polypharmacy, two main approaches have been described: interventions during the prescription process, mainly with the use of computerised physician order entry linked to clinical decision support systems based on practice guidelines; and postprescription review processes, with or without computerised support. Preventive interventions are desired so that clinicians, at the point of prescribing, should prescribe after making an holistic assessment of the patient rather than a single presenting disease, so that the factors raised in the review process are taken into consideration. ${ }^{11}$ However, the complexity of healthcare systems with the intervention of multiple prescribers may necessitate interventions that are based on deprescribing interventions or review of appropriate polypharmacy. ${ }^{11}{ }^{12}$ Medication reviews, using explicit or implicit criteria, are suggested as effective interventions to reduce inappropriate polypharmacy. ${ }^{13}$ The majority of these interventions are described in research articles, but their actual implementation as national or regional policies seems to be limited across Europe. In Scotland, a programme to review appropriate polypharmacy has been implemented nationally by developing clinical guidance and building an economic case that would ensure sustainability for healthcare managers and policy makers. ${ }^{9}$ A key feature was that workforce sustainability was also considered, and an innovative model of pharmacists undertaking reviews with doctors was deployed. The guidance (http://www.polypharmacy. scot.nhs.uk/) builds on the economic case, with data collected during the reviews that demonstrated that the number of medicines was reduced but also that the medicines reduced were high-risk medicines (ie, those more likely to cause admission to hospital). ${ }^{14} 15$ The Scottish polypharmacy guidelines were developed by geriatricians, pharmacists and general practitioners from both hospital and community settings and consist of a seven-step process, which includes an holistic assessment of indication, determination of effectiveness, checking of side effects and numbers needed to treat, and includes a discussion with the patient about their medications. These reviews are mostly undertaken by pharmacists and physicians in the community, in general practice and nursing homes, but clinicians from hospitals (physicians and pharmacists) also undertake work in the community. Patients are prioritised for review on the basis of risk of harm from medications, risk-stratifying by number of medications and risk of readmission to hospital using a national database. Additional partner countries in the SIMPATHY Consortium undertaking review of appropriate polypharmacy in the hospital setting include. Sweden, Naples, Catalonia and Northern Ireland.

Considering the need to improve polypharmacy management across Europe, and with the objective of learning from each other, a European project was created. The SIMPATHY Project, 'Stimulating Innovation Management of Polypharmacy and Adherence in the Elderly', is funded by the European Commission through EU Health Programme 2014-2020. The project is being delivered by a consortium of 10 institutions from eight European countries. The objective of SIMPATHY is 'to stimulate and support innovation across 
the EU in the management of polypharmacy and adherence in the elderly, with specific focus on addressing inappropriate polypharmacy'. To achieve this goal, the SIMPATHY Project has established a series of activities.

1. To describe the need, vision and ambitions for addressing this public health issue in terms of patient safety and reducing harm while optimising outcomes from medicines for citizens across the EU.

2. To produce case studies illustrating the range of development status of polypharmacy and adherence management programmes in the elderly in different EU member states within the consortium.

3. To benchmark current healthcare models and practices for management of inappropriate polypharmacy and non-adherence across the EU.

4. To develop knowledge-sharing networks and resources to support policymakers and healthcare providers through the innovation and change management process to realise best practice, utilising a multidisciplinary team that includes a pharmacist.

5. To exchange best practice ideas on management of appropriate polypharmacy and adherence in the elderly population.

6. To address barriers to innovation in multidisciplinary healthcare provision in the context of management of polypharmacy.

7. To support the dissemination of evidence-based care models, change management tools and expertise, and use best practice to support implementation.

8. To influence and drive change in healthcare practice and policy to deliver better health outcomes from medicine use in the elderly across the EU.

During its first year, SIMPATHY compiled nine case studies that show the current status of six polypharmacy management programmes, and three others that analyse the situation in countries with no programme implemented. The information provided in each of these case studies was obtained through a three-step process: an initial desk review to produce a first insight into the environment; followed by a series of in-depth interviews with relevant informants analysed with qualitative research techniques; ending with focus groups that allow triangulation of the themes that emerge in the two previous steps.

In parallel, an extensive literature review of published and grey documents was performed. This exercise aimed to gather all the existing guidelines on polypharmacy management in Europe.

The next step in the SIMPATHY agenda is a European benchmarking survey that will provide major insight into the details of the existing polypharmacy management programmes across the EU, which will also help to raise awareness of what might be expected from these programmes in countries without them, as well as to determine the kind of data that are collected, or should be collected, to enable implementation of programmes in practice.

To enable the implementation of polypharmacy programmes in Europe and to facilitate the use of all the information gathered in the three previous activities, a tool box to guide strategic decisionmaking will also be created by means of a PESTEL (political, economic, sociocultural, technological, environment and legal) analysis.

Each of these elements will be used to develop a strategy to manage appropriate polypharmacy and adherence in the EU, but also to inform a European polypharmacy handbook, which will be available to all countries across the EU, containing tools that will help countries with different healthcare systems to implement processes to achieve this.

We should be aware that deprescribing cannot be an automated robotic task. We should also be aware that isolated deprescribing practices may not be the best solution for patients and populations. ${ }^{6} 1316$ Thus, the final goal of the SIMPATHY Project is to raise awareness and to create conditions that help different target groups (from policymakers and healthcare providers to caregivers and patients' associations) to identify and implement the best practices for managing polypharmacy in the elderly in the EU.

\section{EAHP Statement 6: Education and Research}

Funding This publication is part of the project 663082/SIMPATHY which has received funding from the European Union's Health Programme (2014-2020).
Competing interests None declared.

Provenance and peer review Not commissioned; externally peer reviewed.

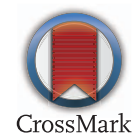

To cite Mair A, Fernandez-Llimos F, SIMPATHY Consortium. Eur J Hosp Pharm 2017;24:5-6.

Eur J Hosp Pharm 2017;24:5-6.

doi:10.1136/ejhpharm-2016-001044

\section{REFERENCES}

1 Barnett K, Mercer SW, Norbury M, et al. Epidemiology of multimorbidity and implications for healthcare, research, and medical education: a cross sectional study. Lancet 2012;380:37-43.

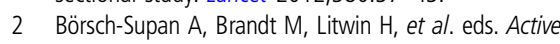
ageing and solidarity between generations in Europe: First results from SHARE after the economic crisis. Berlin: De Gruyter, 2013. ISBN 978-3-11-029546-7.

3 Payne RA, Avery AJ. Polypharmacy: one of the greatest prescribing challenges in general practice. Brit J Gen Prac 2011;61:83-4.

4 Payne RA, Avery AJ, Duerden M, et al. Prevalence of polypharmacy in a Scottish primary care population. Eur I Clin Pharmacol 2014;70:575-81.

5 Gurwitz JH, Field TS, Harrold LR, et al. Incidence and preventability of adverse drug events among older persons in the ambulatory setting. JAMA 2003;289:1107-16.

6 Spinewine A, Schmader KE, Barber $\mathrm{N}$, et al. Appropriate prescribing in elderly people: how well can it be measured and optimised? Lancet 2007;370:173-84.

7 Duerden M, Avery T, Payne R. Polypharmacy and medicines optimisation. England: Kings Fund, 2013.

8 Patterson SM, Cadogan CA, Kerse N, et al. Interventions to improve the appropriate use of polypharmacy for older people. Cochrane Database Syst Rev 2014;(10):CD008165.

9 Johnell K, Klarin I. The relationship between number of drugs and potential drug-drug interactions in the elderly: a study of over 600,000 elderly patients from the Swedish Prescribed Drug Register. Drug Saf 2007;30:911-8.

10 Ferreira JM, Galato D, Melo AC. Medication regimen complexity in adults and the elderly in a primary healthcare setting: determination of high and low complexities. Pharm Pract (Granada) 2015;13:659.

11 Model of Care Group. Scottish polypharmacy guidance. Scottish Government, 2015.

12 Cooper JA, Cadogan CA, Patterson SM, et al. Interventions to improve the appropriate use of polypharmacy in older people: a Cochrane systematic review. BMJ Open 2015;5:e009235.

13 Jansen J, Naganathan V, Carter SM, et al. Too much medicine in older people? Deprescribing through shared decision making. BMJ 2016;353:i2893.

14 Mair A, Keel A. Polypharmacy Guidance for the safe and effective use of multiple medicines to manage long term conditions. http://www.sehd.scot.nhs.uk/ dl/DL(2015)04.pdf (accessed 4 Oct 2016).

15 Pirmohamed M, James S, Meakin S, et al. Adverse drug reactions as cause of admission to hospital: prospective analysis of 18820 patients. BMJ 2004:329:15-9.

16 Page AT, Etherton-Beer CD, Clifford RM, et al. Deprescribing in frail older people-Do doctors and pharmacists agree? Res Social Adm Pharm 2016;12:438-49. 